\title{
Development and characterization of novel 2'-F-RNA aptamers specific to human total and glycated hemoglobin
}

Anna Davydoval, Mariya Vorobyeva, Eugenia Bashmakova, ${ }^{1,2}$, Pavel Vorobjev ${ }^{1,3}$, Olga Krasheninina ${ }^{1}$, Alexey Tupikin ${ }^{1}$, Marsel Kabilov ${ }^{1}$, Vasilisa Krasitskaya $^{1,2}$, Ludmila Frank ${ }^{2,4}$, Alya Venyaminova ${ }^{1}$

${ }^{1}$ Institute of Chemical Biology and Fundamental Medicine SB RAS, Novosibirsk, Russia

${ }^{2}$ Institute of Biophysics SB RAS, Federal Research Center "Krasnoyarsk Science Center SB RAS", Krasnoyarsk, Russia

${ }^{3}$ Department of Natural Sciences, Novosibirsk State University, Pirogova St., 2, 630090 Novosibirsk, Russia

${ }^{4}$ Siberian Federal University, Krasnoyarsk 660041, Russia

*Corresponding author, e-mail anna.davydova@niboch.nsc.ru, tel. +73833635129

\begin{abstract}
Aptamers are short DNA and RNA fragments which bind their molecular targets with affinity and specificity comparable to those of antibodies. Here, we describe the selection of 2'-F-RNA aptamers against total human hemoglobin or its glycated form HbA1c. After SELEX and high-throughput sequencing of the enriched libraries, affinities and specificities of candidate aptamers and their truncated variants were examined by the solid-phase bioluminescent assay. As a result, we identified aptamers specific to both hemoglobins or only glycated HbA1c. The developed 2'-F-RNA aptamers have shown their applicability for detection of total and glycated hemoglobin in one sample using the solid-phase sandwich assay.
\end{abstract}

\section{Highlights}

- Selected new 2'-F-RNA aptamers to total and glycated human hemoglobins

- Truncated aptamer candidates and examined their affinities to choose the best binders

- Demonstrated a proof of principle for detection of total and glycated hemoglobin in one sample using the developed 2'-F-RNA aptamers

\section{Introduction}


Aptamers are relatively short fragments of DNA or RNA which bind their molecular targets with high affinity and specificity. Their binding characteristics are comparable to those of monoclonal antibodies, at that aptamers possess several unique advantages such as a possibility of chemical synthesis with minimal batch-to-batch variation, long shelf-life, stability in a wide range of conditions, and a large variety of available chemical modifications. Aptamers are generated by in vitro selection procedure which means that no immunization of animals is necessary, and any molecule is suitable as a target, regardless of its size or toxicity. Therefore, aptamers represent now a most potent alternative to monoclonal antibodies. A great number of studies have been published to the moment on development and applications of aptamers for therapeutic targets, including modulating the properties of specific proteins [1,2] or targeted drug delivery $[3,4]$. Another large field of aptamers' application is the development of detection systems for different analytes, especially those of diagnostic value. A possibility to modify aptamers in a number of ways and to build them as parts into analytical systems at option gave rise to the enormous diversity of aptamerbased biosensors (aptasensors) with different types of detection and strategies of enhancing the assay performance (see, e.g. the reviews [5-7]).

In a present study, we aimed to develop 2'-F-modified RNA aptamers suitable for detection of glycated human hemoglobin, one of the most important diagnostic markers of diabetes mellitus [8]. Several 2'-F-RNA aptamers binding either total hemoglobin or its glycated form HbA1c were selected and successfully tested in model solid-phase sandwich-type bioluminescent assay.

\section{Materials and methods}

Materials and reagents. Streptavidin, human hemoglobin $(\mathrm{Hb})$ and human serum albumin (HSA) were from Sigma-Aldrich (USA), human glycated hemoglobin (HbA1c) - from Fitzgerald (USA). Polyclonal anti-Hb antibodies - from Novus Biologicals (USA). Ultramild $\beta$-cyanoethyl phosphoramidites and corresponding polymer support were from GlenResearch (USA). 2'-Fmodified pyrimidine nucleoside triphosphates were from Nanotech-C (Russia). Succinimidyl-4-(Nmaleimidomethyl)-cyclohexane-1-carboxylate (SMCC), phosphate-buffered saline pH 7.0-7.3 (PBS), T7 RNA polymerase, inorganic pyrophosphatase, DNase I, RevertAid reverse polymerase, FastAP Cthermosensitive alkaline phosphatase, tosyl activated M-280 Dynabeads ${ }^{\mathrm{TM}}$, T4 polynucleotide kinase, and unmodified ribonucleoside triphosphates were purchased from Thermo Fisher Scientific (USA). Taq DNA polymerase and deoxyribonucleoside triphosphates were purchased from Biosan (Russia).

The highly purified $\mathrm{Ca}^{2+}$-regulated photoprotein obelin with a unique cysteine residue (Obe D12C) was obtained as described previously [9]. 
A solid-phase bioluminescent assay was carried out in white 96-well opaque polystyrene plates (Corning, USA).

Immobilization of target proteins on magnetic beads. $\mathrm{Hb}$ or $\mathrm{HbA} 1 \mathrm{c}(100 \mu \mathrm{g}, 250 \mu \mathrm{L})$ was mixed with $5 \mathrm{mg}$ of tosyl-activated M-280 Dynabeads $^{\mathrm{TM}}$ and incubated in $0.1 \mathrm{M}$ borate buffer ( $\mathrm{pH} 9.5$ ) overnight at $37{ }^{\circ} \mathrm{C}$ with gentle shaking. The beads were incubated in $1 \mathrm{~mL}$ of PBS, $0.05 \%$ Tween 20, $1 \mathrm{mM}$ EDTA (PBSTE) for $1 \mathrm{~h}$ at $37{ }^{\circ} \mathrm{C}$ with gentle shaking. Hemoglobin-functionalized magnetic beads were washed twice with PBSTE $(1 \mathrm{~mL})$ and resuspended in $250 \mu \mathrm{L}$ of PBSTE to achieve the final concentration of $20 \mathrm{mg} / \mathrm{mL}$.

To control protein immobilization efficiency, protein-coupled beads $(25 \mu \mathrm{L})$ were incubated with $1.25 \mu \mathrm{g}$ of polyclonal anti-hemoglobin antibodies in PBSTE for $2 \mathrm{~h}$ at $25^{\circ} \mathrm{C}$ with tilting, then washed three times with PBSTE. Then $20 \mu \mathrm{L}$ of SDS-PAGE reducing sample buffer was added, heated at $90{ }^{\circ} \mathrm{C}$ for $10 \mathrm{~min}$. Eluted proteins were analyzed by SDS-PAGE followed by Coomassie staining.

\section{In vitro selection of 2'-F-modified RNA aptamers}

Selection of aptamers specific for $\mathrm{Hb}$. For each round of selection, $10 \mu \mathrm{M}$ modified RNA library (1 nmol) was folded in PBSE buffer by heating to $90{ }^{\circ} \mathrm{C}$ for $5 \mathrm{~min}$ followed by cooling at $25{ }^{\circ} \mathrm{C}$ for 10 min, then supplemented with $0.05 \%$ Tween $20,0.01 \%$ HSA and $100 \mu \mathrm{g} / \mathrm{mL}$ total yeast RNA and incubated for another10 min. Each selection round started with a negative selection step: RNA library was tilted with Tween 20 blocked (rounds 1-5) or HSA-coupled (rounds 6-10) magnetic beads for $1 \mathrm{~h}$ at $25{ }^{\circ} \mathrm{C}$. After magnetic separation, unbound RNAs were collected and incubated with Hb-coupled beads $(0.5 \mathrm{mg})$ for $1 \mathrm{~h}$ at $25{ }^{\circ} \mathrm{C}$ with tilting. Beads were washed with PBSTE $(200 \mu \mathrm{L})$ three times, suspended in water $(20 \mu \mathrm{L})$ and heated to $95{ }^{\circ} \mathrm{C}$ for $5 \mathrm{~min}$ to elute bound RNAs. Retrieved RNAs were reverse transcribed using reverse PCR primer and RevertAid reverse transcriptase. The cDNA PCR amplification using forward and reverse primers was followed by T7 RNA transcription with $1 \mathrm{mM}$ ATP, GTP and $3 \mathrm{mM}$ 2'-F-UTP, 2'-F-CTP, and T7 RNA polymerase. Transcribed RNA library was purified by gel-filtration and used for next selection round. The stringency of selection was increased by decreasing incubation time and amount of target protein and increasing of washing numbers.

Selection of aptamers specific for HbAlc. In general, the SELEX procedure was performed according to the protocol described above. For aptamer selection, HbA1c was attached to tosyl activated magnetic beads and used as a target for positive selection step. The negative selection included incubation with Tween 20 blocked magnetic beads for rounds 1-7, and Hb-coupled magnetic beads for rounds 8-12. 
DNA melt assay. To assess the diversity of the libraries during the selection, the DiStRO assay [10] was applied. A probes of $0.5 \mu \mathrm{M}$ dsDNA, 1x SYBR Green I, $10 \mathrm{mM}$ sodium cacodylate ( $\mathrm{pH}$ 7.4), $100 \mathrm{mM} \mathrm{NaCl}, 20 \mu \mathrm{l}$ in total, were taken in triplicate. Probes were run on the Rotor-Gene Q machine (Qiagen), using the following protocol: hybridization $\left(95^{\circ} \mathrm{C}\right.$ to $35^{\circ} \mathrm{C}, 0.5^{\circ} \mathrm{C} / \mathrm{min}$ ), hold $35^{\circ} \mathrm{C}$ for 1 $\mathrm{h}$, denaturation $\left(35^{\circ} \mathrm{C}\right.$ to $95^{\circ} \mathrm{C}, 0.5{ }^{\circ} \mathrm{C} / \mathrm{min}$, recorded). Triplicates of raw melting curves were averaged; fluorescence of control solution (w/o DNA) was subtracted. Resulting curves were plotted as fluorescence intensity (I) or its derivative (dI/dT) vs temperature.

Illumina high-throughput sequencing and data analysis. Enriched dsDNA libraries $(1 \mu \mathrm{g})$ were reamplified with the primers containing adapters and barcodes for high-throughput Illumina sequencing. The libraries were sequenced on a MiSeq platform using 2x300 bp paired-ends sequencing kit (Illumina) in Genomics Core Facility (ICBFM SB RAS, Novosibirsk, Russia). Raw sequences were analyzed with UPARSE pipeline [11] using Usearch v10.0.240. The UPARSE pipeline included the merging of paired reads; read quality filtering; length trimming; merging of identical reads (dereplication); discarding singleton reads; removing chimeras and OTU clustering using the UNOISE [12] algorithm.

Analysis of predicted structures of individual 2'-F-RNA aptamers. Predicted secondary structures of aptamers were calculated using Vienna RNAfold algorithm [13]. According to selection conditions, we applied a rescaling of energy parameters to $25{ }^{\circ} \mathrm{C}$ and included $\mathrm{G}-$ quadruplex formation into the structure prediction algorithm.

Synthesis of biotinylated aptamers. Freshly prepared solution of NHS-biotin in DMF (15 mM, 50 $\mu \mathrm{L})$ was mixed with $7 \mathrm{nmol}$ of $\mathrm{NH}_{2}$-modified oligonucleotide in $50 \mu \mathrm{L} 0.1 \mathrm{M} \mathrm{Na}_{2} \mathrm{~B}_{4} \mathrm{O}_{7}$ and incubated for $3 \mathrm{~h}$ at $25{ }^{\circ} \mathrm{C}$. Biotinylated aptamers were precipitated by acetone and then purified using Amicon Ultra-0.5 mL centrifugal filter (Millipore, USA).

Synthesis of obelin conjugate with the anti-Hb antibodies. Anti-Hb antibodies was incubated with SMCC (70-fold molar excess) for $2 \mathrm{~h}$ at $25^{\circ} \mathrm{C}$ in $50 \mathrm{mM}$ 2-(bis(2-hydroxyethyl)aminoacetic acid (BICINE), pH 8.5, 5 mM EDTA. The reagent excess was removed by gel filtration. The SMCCactivated antibody was incubated with Obe D12C (20-fold molar excess) in $20 \mathrm{mM}$ Tris-HCl, pH 7.0, $5 \mathrm{mM}$ EDTA overnight at $40{ }^{\circ} \mathrm{C}$. The synthesized conjugate was purified by gel filtration on Superdex 200 column (GE Healthcare), equilibrated with $20 \mathrm{mM}$ Tris-HCl, pH 7.0, and supplemented with $5 \mathrm{mM}$ EDTA and $0.15 \mathrm{M} \mathrm{NaCl}$.

Solid-phase bioluminescent assay. For the bioluminescent assay, each RNA aptamer was refolded by heating to $90^{\circ} \mathrm{C}$ for $5 \mathrm{~min}$ in PBS with $1 \mathrm{mM} \mathrm{MgCl} 2$ (PBSM) followed by cooling at $25^{\circ} \mathrm{C}$ for 10 min. Folded RNA aptamer was then supplemented with $0.05 \%$ Tween 20, $0.01 \%$ HSA and $100 \mu \mathrm{g} / \mathrm{mL}$ total yeast RNA and incubated at $25^{\circ} \mathrm{C}$ for $10 \mathrm{~min}$. 


\section{Affinity evaluation of 2'-F-RNA aptamers}

Streptavidin solution was placed into the wells $\left(75 \mu \mathrm{L}, 10 \mu \mathrm{g} / \mathrm{mL}\right.$, in PBS) incubated for $1 \mathrm{~h}$ at $37^{\circ} \mathrm{C}$ and washed with PBSM containing $0.05 \%$ Tween 20 (PBSTM). Then biotinylated RNA aptamer (50 $\mu \mathrm{L}, 100 \mathrm{nM}$ in PBSM) was added, incubated at $25^{\circ} \mathrm{C}$ for $1 \mathrm{~h}$, and washed. $50 \mu \mathrm{L}$ aliquots of $\mathrm{Hb}$ $(0.8-26.2 \mu \mathrm{M})$ or HbA1c $(0.12 \mathrm{nM}-2 \mu \mathrm{M})$ in PBSTM were added into the wells and shaken for an hour at $25^{\circ} \mathrm{C}$. After washing, the conjugate of obelin with the anti-Hb antibody $(75 \mu \mathrm{L}, 0.1 \mu \mathrm{M}$, in PBSM) was added, incubated for $40 \mathrm{~min}$ at $25^{\circ} \mathrm{C}$ and washed thereafter. Obelin bioluminescence was measured with LB 940 Multimode Reader Mithras (Berthold, Germany) by rapid injection of 0.1 $\mathrm{M} \mathrm{CaCl}_{2}$ in $0.1 \mathrm{M}$ Tris-HCl, $\mathrm{pH} 8.8$ into the well. The signal was integrated for $5 \mathrm{~s}$.

Bioluminescence intensities from the wells containing incubation buffer only (control samples) were subtracted from signals of the wells with hemoglobins. All experiments were carried out in duplicate. The values of the equilibrium dissociation constant $\left(\mathrm{K}_{\mathrm{D}}\right)$ were determined by approximation of experimental data using a standard equation for bimolecular ligand-receptor binding using the GraphPad Prism software.

Hemoglobins detection in model samples.

Biotinylated aptamer H9t1 (wells A) or gH1t (wells B) (100 nM, $50 \mu \mathrm{L})$ placed into streptavidinactivated wells, incubated with shaking at $25{ }^{\circ} \mathrm{C}$ for $1 \mathrm{~h}$, then washed. Solutions of total $\mathrm{Hb}$ in aptamer buffer $(2 \mu \mathrm{M}, 50 \mu \mathrm{L})$ containing $0,15,7.5,3.75 \% \mathrm{HbA} 1 \mathrm{c}$, were placed into the wells, incubated and washed, then the conjugate of obelin with anti-Hb antibody (50 $\mu \mathrm{L}, 0.1 \mu \mathrm{M}$, in PBSM) was added, incubated for $40 \mathrm{~min}$ at room temperature and washed thereafter. Obelin bioluminescence was measured as described above. Wells without aptamers were processed in the same way and taken as controls. All experiments were carried out in duplicate.

\section{Results and discussion}

In vitro selection of 2'-F-modified RNA aptamers against total and glycated human hemoglobins

To separate bound and unbound RNA molecules during the SELEX procedure, we immobilized target proteins on the surface of tosyl-activated magnetic beads (M-280 Dynabeads). Total or glycated $\mathrm{Hb}$ was coupled via the reaction of tosyl groups on the beads' surface with the amino groups of the proteins. Immobilization was verified using anti-Hb antibodies that bind both total and glycated $\mathrm{Hb}$. After incubation, bound antibodies were eluted by SDS-PAGE reducing sample buffer. Electrophoretic analysis of supernatant samples showed bands characteristic to antibodies (see Supplementary data, Fig. S1). Moreover, this experiment also proved that bead-coupled proteins maintained their native structure after the immobilization procedures. 
In order to obtain aptamers which bind total $\mathrm{Hb}$, we used a commercial protein (Sigma-Aldrich) isolated from healthy donors' blood. According to the manufacturer's description, this protein was isolated as total blood hemoglobin and therefore consists mostly of non-glycated $\mathrm{Hb}$ with a small fraction of glycated forms. Briefly, an initial 2'-F-RNA library consisting of about $10^{15}$ different sequences was incubated with total $\mathrm{Hb}$-modified beads, unbound RNAs were separated and bound RNAs were eluted from beads and amplified by RT-PCR followed by T7 transcription of RNA library (Fig. 1). To exclude bead-binding sequences from RNA pool, we performed a counter selection step using magnetic beads blocked by Tween 20 , starting from round 2 . To eliminate nonspecific aptamers, we used bead-immobilized HSA for counter selection step at rounds 7-10.

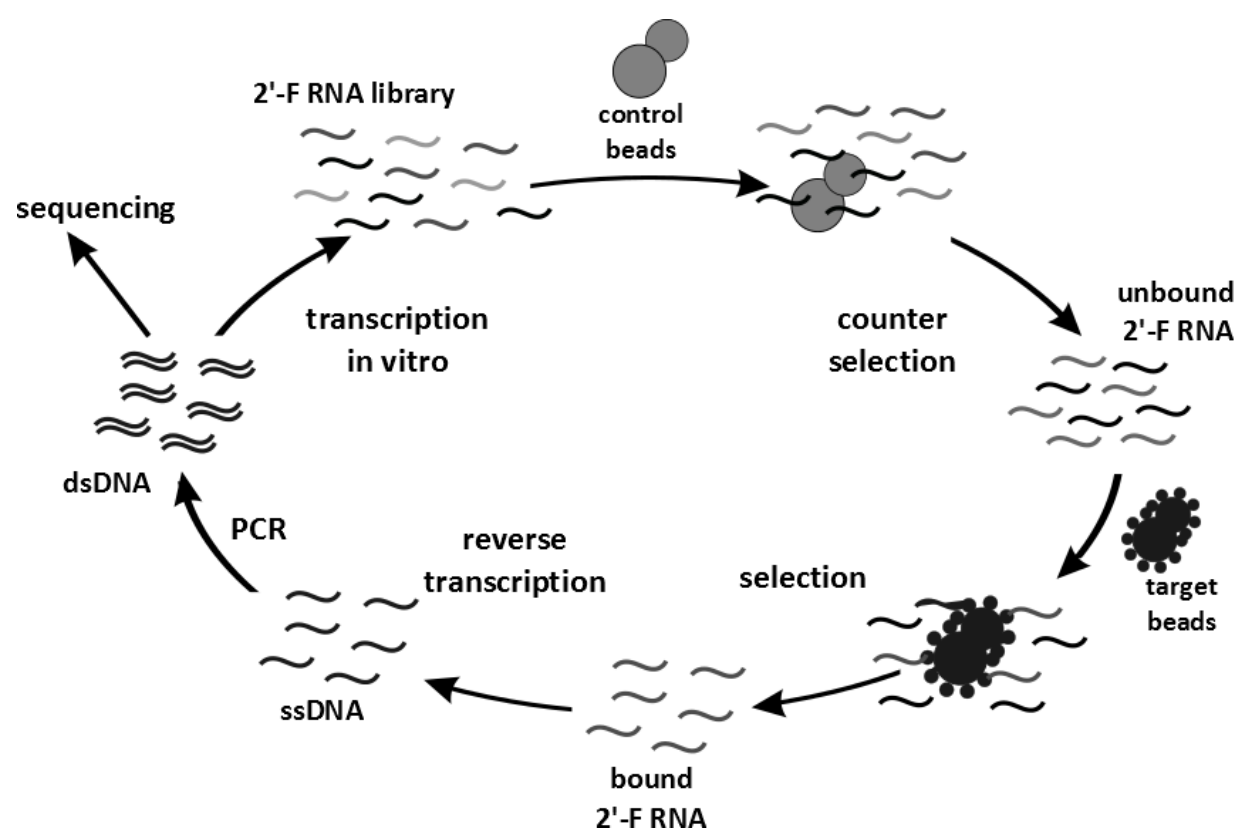

Figure 1. In vitro selection of 2'-F-RNA aptamers using magnetic bead-immobilized total or glycated $\mathrm{Hb}$. Counter selection steps included incubations with Tween-blocked beads, HSAmodified beads (for aptamers to total $\mathrm{Hb}$ ) or $\mathrm{Hb}$-modified beads (for aptamers to $\mathrm{HbA1c}$ ).

The selection pressure was progressively increased by decreasing the amount of protein-coupled magnetic beads and the time of incubation and increasing the number of washings. To improve the binding affinity of the enriched library, after the positive selection step we washed RNA-bound magnetic beads with PBSTE buffer supplemented with $0.5 \mathrm{M} \mathrm{NaCl}$ or $0.5 \mathrm{M}$ urea at the $9^{\text {th }}$ and $10^{\text {th }}$ rounds, respectively. According to [14], these agents favor discarding low-affine sequences from the enriched pool.

To select RNA aptamers to glycated hemoglobin HbA1c, we performed analogous SELEX procedure using HbA1c-coupled magnetic beads as a target. Counter selection steps included incubations with Tween-blocked beads for rounds 1 through 7 and with total Hb-coupled magnetic 
beads from the $8^{\text {th }}$ round, to obtain aptamers specific to glycated protein form. We also used washing buffer with high salt concentration $(0.5 \mathrm{M} \mathrm{NaCl})$ to exclude weak binders. After each SELEX round, the enrichment of the pool was evaluated by analyzing RT-PCR products of negative and positive selections (see, e.g., Fig. 2).

A

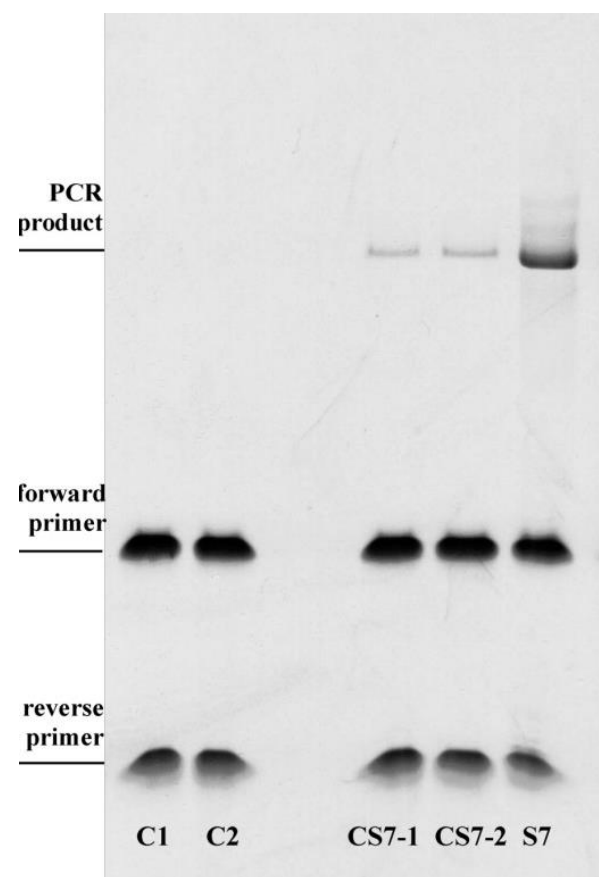

B

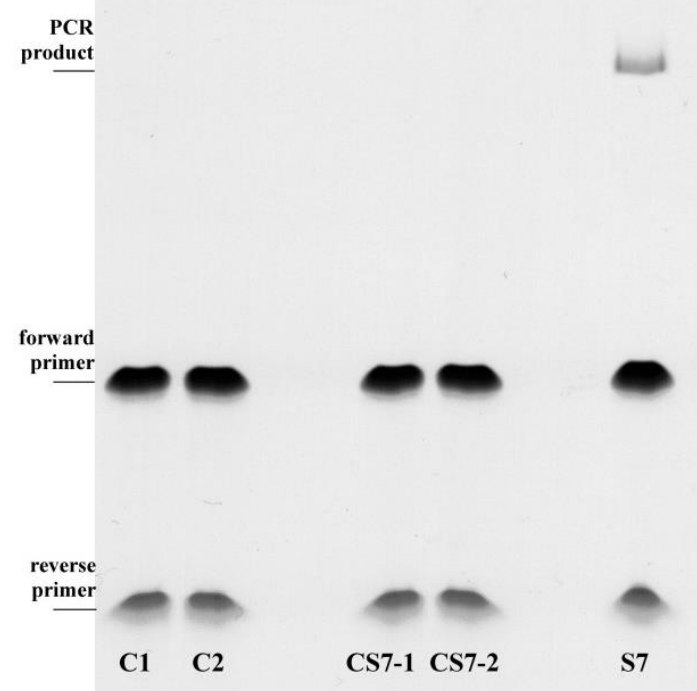

Figure 2. Electrophoretic analysis of enriched DNA libraries after PCR amplification at the $7^{\text {th }}$ round of SELEX to total (A) and glycated (B) Hb using 12\% denaturing PAAG. Lanes C1 and C2 controls without templates; lanes CS7-1 and CS7-2- products of counter selection to HSA-coupled beads (A) and Tween-blocked beads (B); lanes S7 - products of positive selection.

To monitor the course of selection, we assessed the diversities of the initial and enriched libraries by analyzing remelting profiles of dsDNA pools (DiStRO method) [10]. The progressive increase of remelting temperature with the number of the round evidenced for the loss of diversity which corresponds to the enrichment of the libraries (Fig. 3).
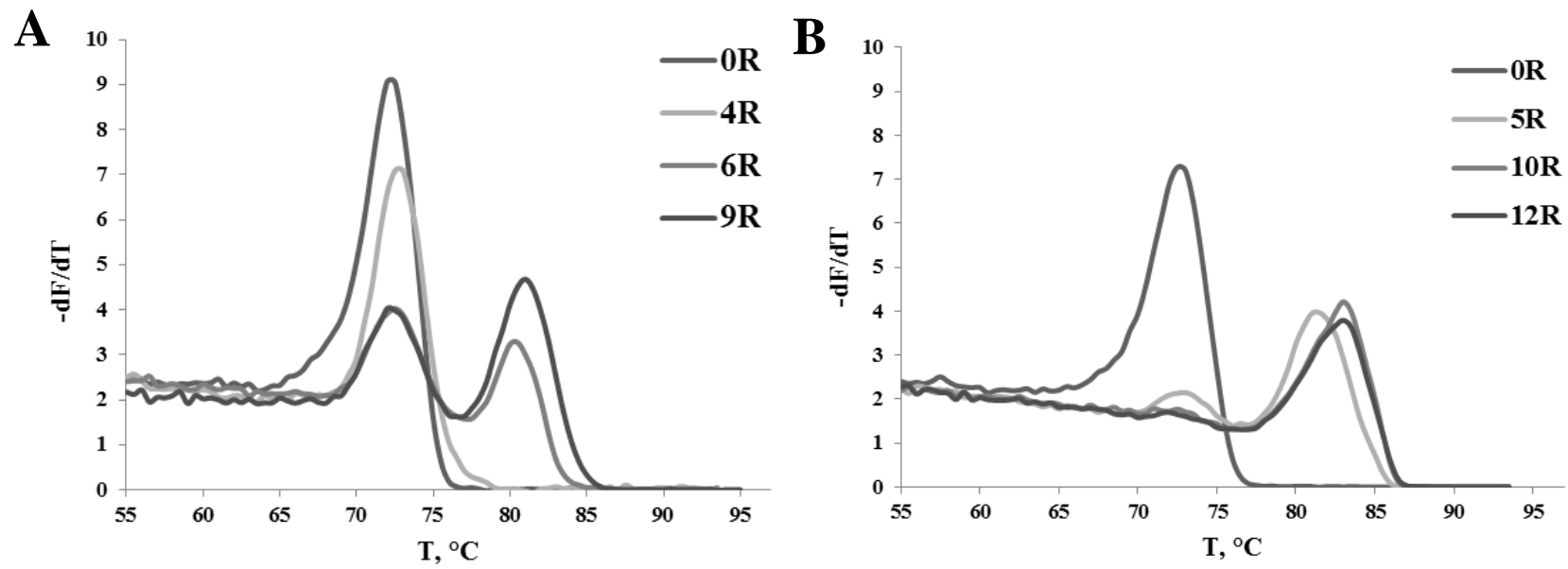
Figure 3. Normalized differential remelting curves of the initial and enriched libraries (in the presence of SYBR Green I). A - the selection of aptamers to total $\mathrm{Hb}, \mathrm{B}$ - the selection of aptamers to $\mathrm{HbA} 1 \mathrm{c}$.

The enriched libraries after $4^{\text {th }}, 8^{\text {th }}$ and $10^{\text {th }}$ SELEX rounds against total hemoglobin and after $4^{\text {th }}, 7^{\text {th }}$ $11^{\text {th }}$ and $12^{\text {th }}$ rounds against glycated hemoglobin were re-amplified with barcode-bearing primers and sequenced with the Illumina NGS. Bioinformatic analysis of NGS data revealed the total number sequence distribution in every pool. All sequences were ranked according to their abundance at the final enriched libraries (see tables S2 and S3 in Supplementary data). For each selection, we further analyzed sequences with $>1 \%$ abundances.

After 10 rounds of selection to total $\mathrm{Hb}$, aptamer $\mathrm{H} 1$ represented $25.5 \%$ of all sequences in the enriched library, with a sequential increase of abundance during the SELEX (table S2). Analysis of predicted secondary structures using VIENNA RNAfold algorithm (see Materials and methods) revealed G-quadruplex (GQ) forming motifs for sequences H5, H7, H5136, and H9. While GQ motifs are quite uncommon for RNA aptamers, several GQ forming RNA aptamers have been previously reported, including those for prion proteins [15,16], human interleukin-6 receptor [17], HIV-1 Tat protein [18], and fluorophore binding Spinach aptamer [19]. GQ motifs could potentially provide a possibility of aptamer regulation due to their cation dependence.

In the case of HbA1c, the final library was mostly enriched by aptamers gH1 (22.5\%), gH2 (10.2\%) and its mutant $\mathrm{gH} 10(6.8 \%)$, and $\mathrm{gH} 4(6.1 \%)$. In contrast, none of them contained GQ forming motifs. However, other selection leaders gH8 (2.7 \%) and gH13 (1.2\%) did contain predicted GQs. Notably, gH8 aptamer had the same nucleotide sequence as H5136 selected on the total Hb. Since its abundance progressively decreased through the rounds with counter selection on total $\mathrm{Hb}$ (Table S3), we hypothesized that gH8 may bind an aptatope common for both glycated and non-glycated proteins.

Thus, we chose aptamers H1, H5, H9, gH1, gH2, gH4 and gH8 as candidates for further studies.

\section{Individual RNA aptamers: truncation and synthesis}

Truncation of candidate aptamers reduces the cost of their production and may increase the binding affinity. To this point, we analyzed aptamers' predicted secondary structures (see Fig. S2). Both constant and random regions of $\mathrm{H} 1$ aptamer were involved in secondary structure formation, so elimination of primer-binding sites may decrease the affinity. In the case of H5 and H9 aptamers, constant regions were less involved in structure formation, so we proposed their trimming. A comparison of secondary structures of full-length and truncated versions of aptamers to $\mathrm{HbA} 1 \mathrm{c}$ 
(gH1, gH2, gH4, and gH8) did not reveal any structural alterations. All candidate aptamers for affinity screening are represented in Table 1.

Table 1. Nucleotide sequences of candidate 2'-F-RNA aptamers.

\begin{tabular}{|c|l|c|}
\hline Aptamer & \multicolumn{1}{|c|}{ Nucleotide sequence, 5'-3' } & $\begin{array}{c}\text { Length, } \\
\text { nt }\end{array}$ \\
\hline H1 & $\begin{array}{l}\text { GGG AGA CAA GAA UAA ACG CUC AAU GGC GCC ACC UAA UGC UCA GUA CUU } \\
\text { AUU UAU GGC GAA CCA UUC GAC AGG AGG CUC ACA ACA GGC }\end{array}$ & 87 \\
\hline $\mathbf{H 5 t 1}$ & $\begin{array}{l}\text { GAC AAG AAU AAA CGC UCA ACC GAG GGA GGG GGG GAG GAU GGC UGU } \\
\text { GCG UUU UAC UUC UUU UC }\end{array}$ & 62 \\
\hline $\mathbf{H 5 t 1 1}$ & AAA CGC UCA ACC GAG GGA GGG GGG GAG GAU GGC UGU GCG UUU & 42 \\
\hline $\mathbf{H 5 t 1 2}$ & CGC UCA ACC GAG GGA GGG GGG GAG GAU GGC UGU GCG & 36 \\
\hline $\mathbf{H 5 t 1 3}$ & CGC CAA CCG AGG GAG GGG GGG AGG AUG GCU GGC G & 34 \\
\hline $\mathbf{H 5 t 1 4}$ & GCC ACC GAG GGA GGG GGG GAG GAU GGU GGC & 30 \\
\hline $\mathbf{H 9 t 1}$ & $\begin{array}{l}\text { CCU GAU CAG CCU GGC AGU CGC GGG GAG GGG GGG AGG AGU UCG ACA GGA } \\
\text { GGC UCA CAA CAG G }\end{array}$ & 61 \\
\hline $\mathbf{H 9 t 1 1}$ & GCC UGG CAG UCG CGG GGA GGG GGG GAG GAG UUC GAC AGG AGG C & 43 \\
\hline $\mathbf{H 9 t 1 2}$ & CGU CGC GGG GAG GGG GGG AGG AGU UCG ACG & 30 \\
\hline $\mathbf{g H 1 t}$ & $\begin{array}{l}\text { ACG CUC AAU GGG GAU UCU GUA GUG GGA AGG GCC GAG CGU ACA AGC CUU } \\
\text { CGA CAG GAG GCU }\end{array}$ & 60 \\
\hline $\mathbf{g H 2 t}$ & $\begin{array}{l}\text { GCU CAA GGG GUU CUU GGU AAG UGG GAG GGC CAG GAG CAA AUC GCC } \\
\text { UGU UCG ACA GGA GGC UCA CAA CAG GC }\end{array}$ & 71 \\
\hline $\mathbf{g H 4 t}$ & $\begin{array}{l}\text { GAA UAA ACG CUC AAC UAG AAG GGG CAA UCG UAG GGG ACG CUA GUG } \\
\text { AGA CGG UUA UUC }\end{array}$ & 57 \\
\hline $\mathbf{g H 8 t}$ & ACG CUC AAG GGG GGG UCU UGU GAG UUA GGG UUA GGG CCG AGC GU & 44 \\
\hline
\end{tabular}

All pyrimidines are 2'-fluoro-modified.

Candidate aptamers bearing 3'-amino group for coupling with biotin were chemically synthesized using corresponding solid-phase support.

\section{Bioluminescent assays of 2'-F-RNA aptamers' affinities}

We examined binding affinities of individual aptamers by the solid-phase bioluminescent sandwich microassay. A bioluminescent photoprotein obelin was chosen as a fast, triggerable, and highly sensitive reporter for the assay [20]. 3'-Biotinylated aptamers were immobilized on the streptavidinactivated surface (thus, employed as capture components of the sandwich) and titrated with total or glycated $\mathrm{Hb}$. Aptamer-protein complexes were visualized using obelin-conjugated anti-hemoglobin antibody. Bioluminescence of the photoprotein was triggered by $\mathrm{Ca}^{2+}$ injection (see Fig. 4). 

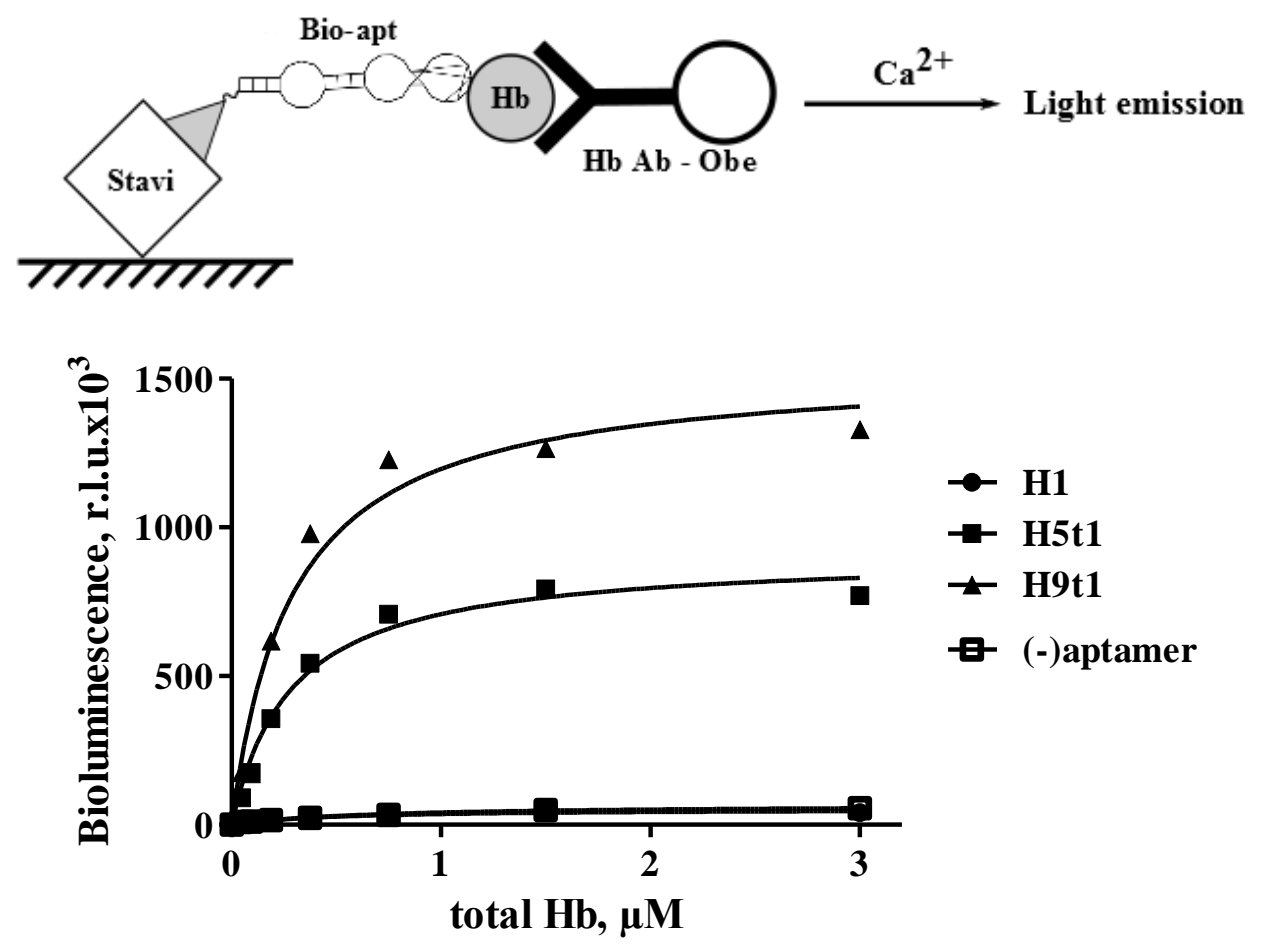

Figure 4. Solid-phase bioluminescent assay of aptamers binding with total $\mathrm{Hb}$. The assay scheme is represented at the top. Stavi - streptavidin; Bio - biotinyl, r.l.u. - relative light units.

Surprisingly, the most abundant $\mathrm{H} 1$ aptamer showed no binding affinity to target total $\mathrm{Hb}$. We supposed this aptamer to be an amplification "parasite" - a nucleotide sequence preferably amplified by PCR. At the same time, aptamers H5t1 and H9t1 with quadruplex-forming motifs sufficiently bound total $\mathrm{Hb}$ with $\mathrm{K}_{\mathrm{D}}$ values of $280 \mathrm{nM}$ and $270 \mathrm{nM}$, respectively (Table 2).

Table 2. $\mathrm{K}_{\mathrm{D}}$ values for binding of 2'-F-RNA aptamers with $\mathrm{Hb}$ and $\mathrm{HbA1c}$.

\begin{tabular}{|c|c|c|}
\hline \multirow{2}{*}{ Aptamer } & \multicolumn{2}{|c|}{$\mathrm{K}_{\mathrm{D}, \mathrm{nM}}$} \\
\cline { 2 - 3 } & $\mathrm{Hb}$ & HbA1c \\
\hline H9t1 & $290 \pm 70$ & $380 \pm 90$ \\
\hline H5t1 & $280 \pm 50$ & $430 \pm 100$ \\
\hline gH8t & $190 \pm 20$ & $110 \pm 40$ \\
\hline gH1t & no binding & $250 \pm 80$ \\
\hline
\end{tabular}

To further minimize the length of these aptamers, we synthesized a series of truncated versions of $\mathrm{H} 5 \mathrm{t} 1$ and H9t1 (table 1) with shortened stems. Bioluminescent analysis of total $\mathrm{Hb}$ binding showed some decrease of affinity for 42-nt H5t11 and almost total loss of affinity after further truncation (H5t12 and H5t13). Truncated 43-nt H9t11 demonstrated the two-time decrease of bioluminescent signal and further truncation (H9t12), again, caused a dramatic loss of binding affinity (Fig. 5). We 
supposed that double-stranded helixes play a critical role for active conformation (probably, for a GQ stabilization) and protein binding of H5t1 and H9t1 aptamers.

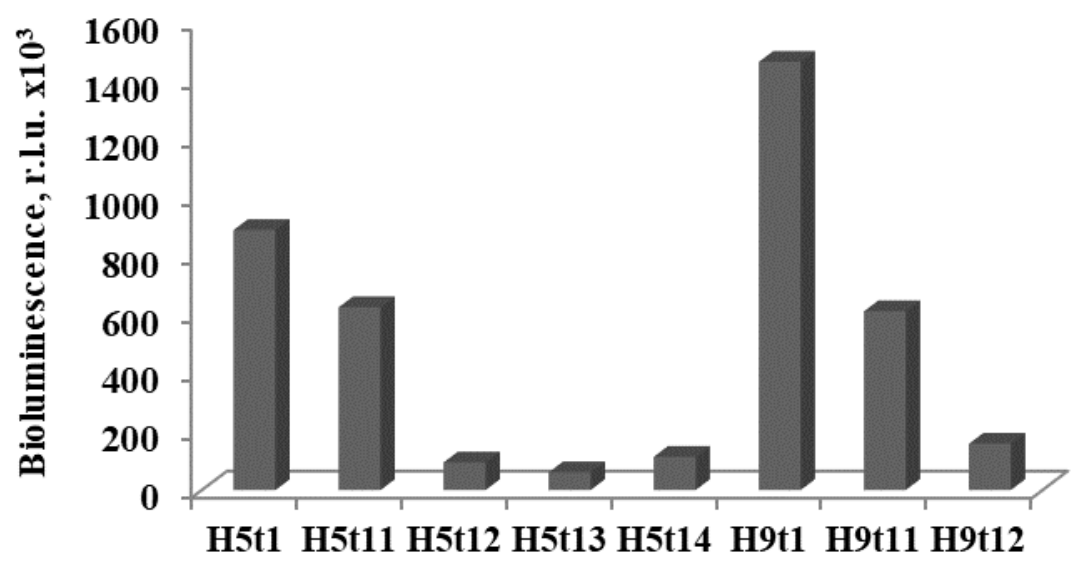

Figure 5. Binding of series of truncated aptamers $\mathrm{H} 5 \mathrm{t} 1$ and $\mathrm{H} 9 \mathrm{t} 1$ with $1 \mu \mathrm{M} \mathrm{Hb}$.

We also analyzed binding of $\mathrm{H} 5 \mathrm{t} 1$ and $\mathrm{H} 9 \mathrm{t} 1$ with glycated hemoglobin HbA1c. According to the results of the solid-phase bioluminescent assay, both aptamers bound glycated form as well as total $\mathrm{Hb}$, with similar $\mathrm{K}_{\mathrm{D}}$ values (Table 2). Therefore, H5t1 and H9t1 aptamers can be regarded as universal recognizing elements for both forms of hemoglobin.

The aptamers selected against glycated hemoglobin (gH1t, gH2, gH4t, and gH8t) were also examined in binding assays with $\mathrm{HbA1c}$ and total $\mathrm{Hb}$ using the same scheme. Interestingly, GQ forming gH8t (H5136) aptamer represented in enriched pools from both selections bound with $\mathrm{HbA1c}$ with nearly the same $\mathrm{K}_{\mathrm{D}}$ value as for total $\mathrm{Hb}$ (Table 2), which confirms the possibility of recognizing some common aptatope within both proteins.

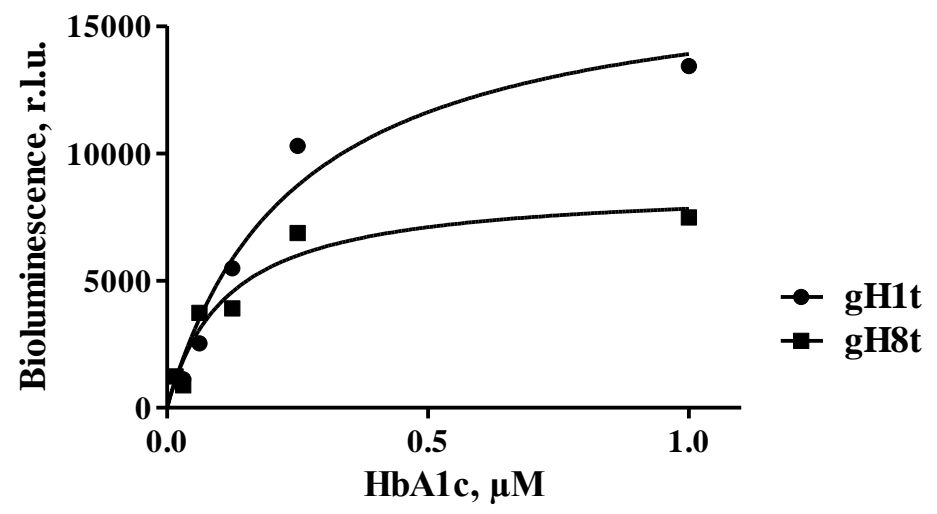

Figure 6. Solid-phase bioluminescent assay of aptamers gH1t and gH8t binding with HbA1c. 
Aptamers $\mathrm{gH} 2 \mathrm{t}$ and $\mathrm{gH} 4 \mathrm{t}$ generated very weak bioluminescent signals for both hemoglobins. The most abundant gH1t aptamer showed the highest bioluminescent signal when titrated by HbA1c (Fig. 6, Table 2), but no binding with the total $\mathrm{Hb}$. Therefore, we consider the aptamer gHt1 as a specific binder of $\mathrm{HbA} 1 \mathrm{c}$.

Comparing 2'-F-RNA aptamers with known DNA aptamers in the bioluminescent sandwich assay

Several DNA aptamers to glycated and total hemoglobins have been reported earlier [21-23]. These DNA aptamers were selected using protein-coupled beads and applied in fluorescent or chemiluminescent systems for detection of $\mathrm{Hb}$ and $\mathrm{HbA1c}[22,23]$. It was of interest to compare our RNA aptamers H9t1 and H5t1 with DNA ones (listed in Table 3) in the same solid-phase bioluminescent sandwich assay. Since the affinity of the aptamer can depend on certain buffer conditions, we examined all DNA aptamers using the same binding buffers as reported in the original papers.

Table 3. DNA aptamers specific to total Hb.

\begin{tabular}{|c|l|c|c|}
\hline Aptamer & \multicolumn{1}{|c|}{ Nucleotide sequence, $\mathbf{5}^{\prime} \mathbf{- 3}^{\prime}$} & $\begin{array}{c}\text { Length, } \\
\text { nt }\end{array}$ & Ref. \\
\hline D10-6 & $\begin{array}{l}\text { GACAGGCAGGACACCGTAACCCGTAGCATTGCGGAAGTCACCGTGGGT } \\
\text { TGAATGGTCTACTGCTACCTCCCTCCTCTTC }\end{array}$ & 79 & {$[23]$} \\
\hline $\mathbf{D 1 0 - 9}$ & $\begin{array}{l}\text { GACAGGCAGGACACCGTAAGCGCGAGTAAGGGTACGTCTAGTAAC } \\
\text { TCCTCGGTACGGTCCTGCTACCTCCCTCCTCTTC }\end{array}$ & 79 & \\
\hline So6 & TTAGCGAGCTGCACACACAATGGACTCGTCATACCGTGCTGTTT & 44 & {$[21]$} \\
\hline $\mathbf{S o 7}$ & ATCTGCAGAATTCGCCCTTGCTGGTGCAGTACACACCCGGCGGG & 44 & \\
\hline $\begin{array}{c}\text { Hb } \\
\text { aptamer }\end{array}$ & $\begin{array}{l}\text { GGCAGGAAGACAAACACCAGGTGAGGGAGACGACGCGAGTGTTAGAT } \\
\text { GGTAGCTGTTGGTCTGTGGTGCTGT }\end{array}$ & 72 & {$[22]$} \\
\hline
\end{tabular}

All DNA aptamers were 5'-biotinylated (see Materials and methods).

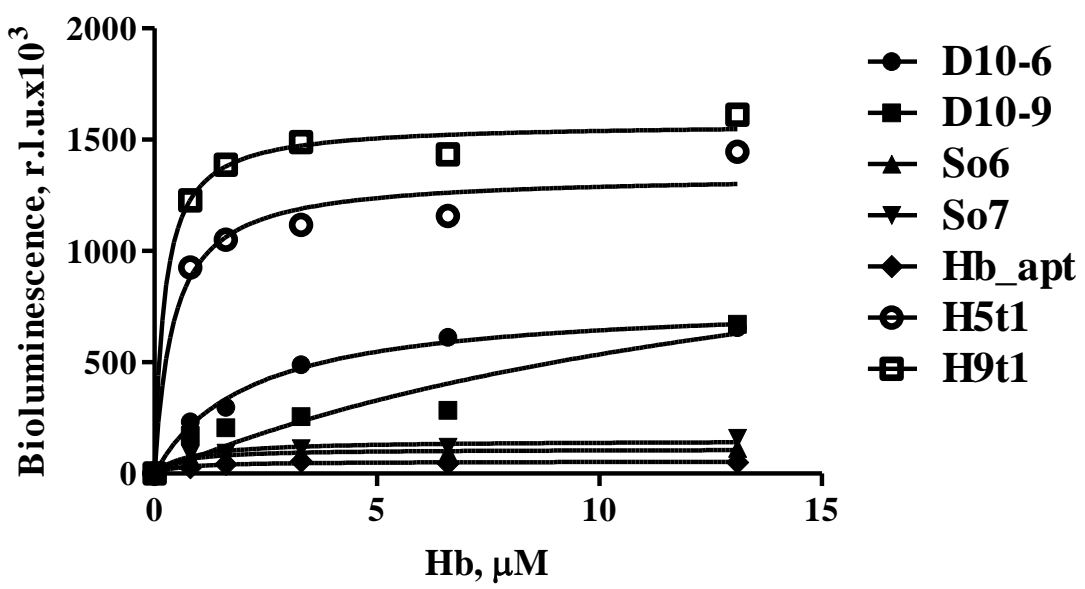

Figure 7. Solid-phase bioluminescent assay of DNA and 2'-F-RNA aptamers binding to total $\mathrm{Hb}$. 
According to results of epybioluminescent assay performed by the scheme described above (Fig. 3, top), DNA aptamers 10-9 and 10-6 reported in [23] demonstrated binding activities comparable to those of 2'-F-RNA aptamers, but with lower signal intensities. The rest of DNA aptamers provided bioluminescent signals close to aptamer-free control points (Fig. 7).

\section{Model bioluminescent hemoglobin assay}

We also employed aptamers $\mathrm{H} 9 \mathrm{t1}$ and gH1t selected to total $\mathrm{Hb}$ and $\mathrm{HbA} 1 \mathrm{c}$, correspondingly, as biospecific elements in model bioluminescent analyses of hemoglobins. To test a possibility of detection of glycated hemoglobin in the presence of large excess of the non-glycated one, we used the solution of $2 \mu \mathrm{M}$ total $\mathrm{Hb}$ (concentration approx. 1000 times less than the physiological one) spiked with $0,15,7.5$ or $3.75 \%$ HbA1c. Aptamers H9t1 (variant A) or gH1t (variant B) immobilized on the surface of microplate wells were incubated with model samples, followed by detection of bound hemoglobins by obelin-antibody conjug (Fig. 8, scheme on the up).

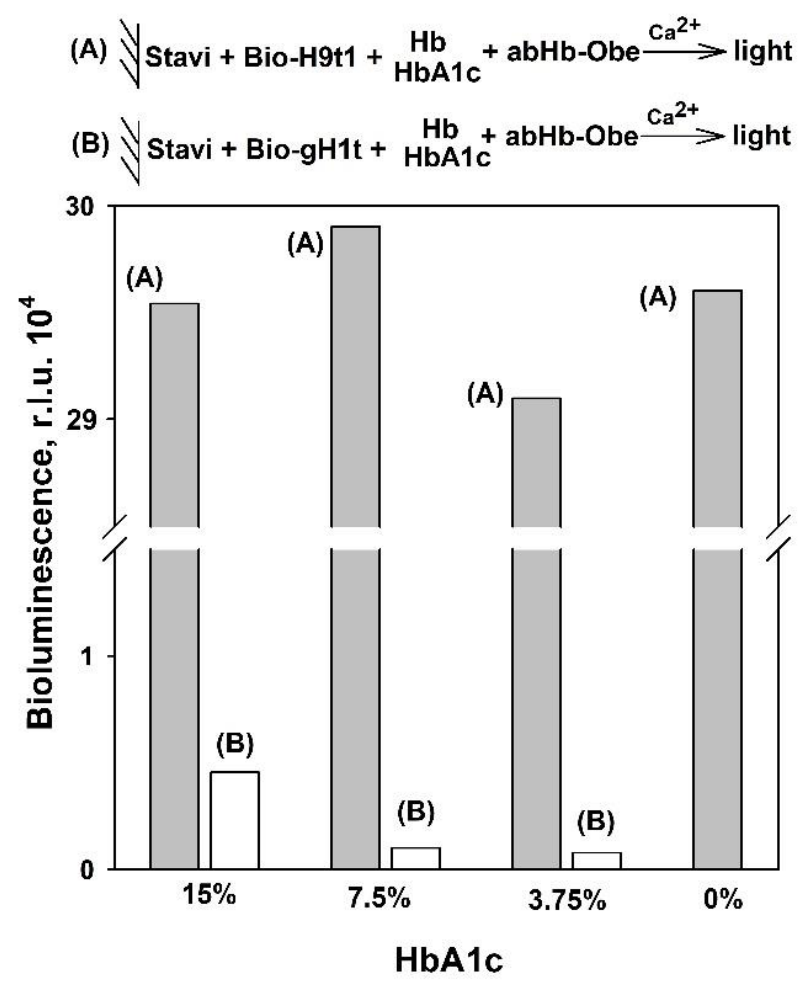

Figure 8. Bioluminescent detection of the total (series A) and glycated (series B) hemoglobins. Stavi - streptavidin; Bio-H9t1 and Bio-gH1t - biotinylated aptamers; abHb-Obe - conjugate anti-Hb antibodies-obelin; r.l.u. - relative light units.

Bioluminescent signals obtained in series A (from total $\mathrm{Hb}$ ) were almost the same (the spread was no more than 3\%). Signals obtained in series B (from HbA1c) decrease in 4 and 1.5 times when target concentrations fall to 7.5 and $3.75 \%$, while signals from the wells without $\mathrm{HbA} 1 \mathrm{c}$ did not differ from 
the control ones. This result demonstrates a proof of principle for detection of total and glycated hemoglobins in a sample using the developed 2'-F-RNA aptamers.

\section{Conclusion}

We selected new 2'-F-RNA aptamers specific to total and glycated human hemoglobin. Interestingly, predicted secondary structures of aptamers to total $\mathrm{Hb}$ contained G-quadruplex forming motifs. At the same time, aptamers specific to glycated hemoglobin consisted of more conventional hairpin elements. We minimized the nucleotide sequences of selected aptamers and examined their affinities in the solid-phase bioluminescent assay. The aptamers selected against total hemoglobin demonstrated almost equal affinities to total and glycated hemoglobin, while the leader of HbA1cSELEX recognized only the glycated protein. Notably, the developed aptamers were found to be applicable as biospecific elements for bioluminescent solid-phase assay of both hemoglobin forms in one sample. Future research will be focused on the development of sandwich-type aptasensor to determine the percent of glycated hemoglobin in blood samples as a long-term glycemic biomarker.

\section{Acknowledgements}

The work was supported by the Russian Science Foundation (grant number 16-14-10296).

\section{Declaration of interest}

The authors have no competing interests to declare. The founding sponsors had no role in the design of the study; in the collection, analysis and interpretation of data; in the writing of the manuscript; nor in the decision to publish the results.

\section{REFERENCES}

[1] S.M. Nimjee, R.R. White, R.C. Becker, B.A. Sullenger, Aptamers as Therapeutics, Annu. Rev. Pharmacol. Toxicol. 57 (2017) 61-79. doi:10.1146/annurev-pharmtox-010716-104558.

[2] Y. Yu, C. Liang, Q. Lv, D. Li, X. Xu, B. Lui, A. Lu, G. Zhang, Molecular Selection, Modification and Development of Therapeutic Oligonucleotide Aptamers, Int. J. Mol. Sci. 17 (2016) 358. doi:10.3390/ijms17030358.

[3] S. Catuogno, C.L. Esposito, G. Condorelli, V. de Franciscis, Nucleic acids delivering nucleic acids, Adv. Drug Deliv. Rev. 134 (2018) 79-93. doi:10.1016/J.ADDR.2018.04.006.

[4] G. Zhou, O. Latchoumanin, L. Hebbard, W. Duan, C. Liddle, J. George, L. Qiao, Aptamers as targeting ligands and therapeutic molecules for overcoming drug resistance in cancers, Adv. Drug Deliv. Rev. 134 (2018) 107-121. doi:10.1016/J.ADDR.2018.04.005. 
[5] T.K. Sharma, J.G. Bruno, A. Dhiman, ABCs of DNA aptamer and related assay development, Biotechnol. Adv. 35 (2017) 275-301. doi:10.1016/j.biotechadv.2017.01.003.

[6] H. Chen, J. Huang, A. Palaniappan, Y. Wang, B. Liedberg, M. Platt, A.I.Y. Tok, A review on electronic bio-sensing approaches based on non-antibody recognition elements, Analyst. 141 (2016) 2335-2346. doi:10.1039/c5an02623g.

[7] H. Zhang, L. Zhou, Z. Zhu, C. Yang, Recent Progress in Aptamer-Based Functional Probes for Bioanalysis and Biomedicine, Chem. - A Eur. J. 22 (2016) 9886-9900. doi:10.1002/chem.201503543.

[8] S. Kojić Damjanov, M. Đerić, N. Eremić Kojić, Glycated hemoglobin A1c as a modern biochemical marker of glucose regulation, Med Pregl. 67 (2014) 339-44.

[9] V. V. Krasitskaya, L.P. Burakova, A.A. Komarova, E.E. Bashmakova, L.A. Frank, Mutants of Ca2+-regulated Photoprotein Obelin for Site-specific Conjugation, Photochem. Photobiol. 93 (2017) 553-557. doi:10.1111/php.12712.

[10] T. Schütze, P.F. Arndt, M. Menger, A. Wochner, M. Vingron, V.A. Erdmann, H. Lehrach, C. Kaps, J. Glökler, A calibrated diversity assay for nucleic acid libraries using DiStRO-a Diversity Standard of Random Oligonucleotides, Nucleic Acids Res. 38 (2009). doi:10.1093/nar/gkp1108.

[11] R.C. Edgar, UPARSE: Highly accurate OTU sequences from microbial amplicon reads, Nat. Methods. 10 (2013) 996-998. doi:10.1038/nmeth.2604.

[12] R.C. Edgar, UNOISE2: improved error-correction for Illumina 16S and ITS amplicon sequencing, BioRxiv. (2016) 081257. doi:10.1101/081257.

[13] A.R. Gruber, R. Lorenz, S.H. Bernhart, R. Neuböck, I.L. Hofacker, The Vienna RNA websuite., Nucleic Acids Res. 36 (2008) 70-74. doi:10.1093/nar/gkn188.

[14] P. Bouvet, Identification of Nucleic Acid High-Affinity Binding Sequences of Proteins by SELEX, Methods Mol Biol. 543 (2009) 139-50. doi:10.1007/978-1-60327-015-1.

[15] T. Mashima, F. Nishikawa, Y.O. Kamatari, H. Fujiwara, M. Saimura, T. Nagata, T. Kodaki, S. Nishikawa, K. Kuwata, M. Katahira, Anti-prion activity of an RNA aptamer and its structural basis, Nucleic Acids Res. 41 (2013) 1355-1362. doi:10.1093/nar/gks1132. 
[16] D. Proske, S. Gilch, F. Wopfner, H.M. Schätzl, E.-L. Winnackera, M. Famulok, Prion-proteinspecific aptamer reduces PrP Sc formation, ChemBioChem. 3 (2002) 717-725. doi:10.1002/1439-7633(20020802)3:8<717::AID-CBIC717>3.0.CO;2-C.

[17] K. Szameit, K. Berg, S. Kruspe, E. Valentini, E. Magbanua, M. Kwiatkowski, I. Chauvot de Beauchêne, B. Krichel, K. Schamoni, C. Uetrecht, D.I. Svergun, H. Schlüter, M. Zacharias, U. Hahn, Structure and target interaction of a G-quadruplex RNA-aptamer, RNA Biol. 13 (2016) 973-987. doi:10.1080/15476286.2016.1212151.

[18] Y. Yamaoki, T. Nagata, T. Mashima, M. Katahira, Development of an RNA aptamer that acquires binding capacity against HIV-1 Tat protein via G-quadruplex formation in response to potassium ions, Chem. Commun. 53 (2017) 7056-7059. doi:10.1039/C7CC03312E.

[19] H. Huang, N.B. Suslov, N.-S. Li, S.A. Shelke, M.E. Evans, Y. Koldobskaya, P.A. Rice, J.A. and Piccirilli, A G-Quadruplex-Containing RNA Activates Fluorescence in a GFP-Like Fluorophore, Nat Chem Biol. 10 (2014) 686-691. doi:10.1038/nchembio.1561.A.

[20] L.A. Frank, Ca2+-regulated photoproteins: Effective immunoassay reporters, Sensors. 10 (2010) 11287-11300. doi:10.3390/s101211287.

[21] D.-S. Cameron, Brent D., Kim, Methods and Devices for Detection and Measurement of Analytes, 1 (2014). doi:US 2014/0335630 A1.

[22] H.I. Lin, C.C. Wu, C.H. Yang, K.W. Chang, G. Bin Lee, S.C. Shiesh, Selection of aptamers specific for glycated hemoglobin and total hemoglobin using on-chip SELEX, Lab Chip. 15 (2015) 486-494. doi:10.1039/c4lc01124d.

[23] M. Lin, W. Li, Y. Wang, X. Yang, K. Wang, Q. Wang, P. Wang, Y. Chang, Y. Tan, Discrimination of hemoglobins with subtle differences using an aptamer based sensing array, Chem. Commun. 51 (2015) 8304-8306. doi:10.1039/c5cc00929d. 